\title{
Spatial Resolution of X-Ray Images
}

\author{
Raynald Gauvin and Pierre Michaud \\ Department of Mining and Materials Engineering, McGill University, 3610 University Street, \\ Montréal, Québec, Canada, H3A 2B2.
}

Current SDD detectors can now deliver 200000 to 1000000 of counts per seconds and the count rates is now limited by the maximum probe current available in the electron microscope for a given probe diameter. The question of imaging materials with x-ray in the SEM needs to be investigated; especially the relation between the spatial resolution and the number of x-ray counts in the x-ray image. This paper will demonstrate how Monte Carlo simulations can be used to determine this relationship using a new Monte Carlo program, MC X-Ray [1].

This new program computes the complete $x$-ray spectra from the simulation of electron scattering in solids with various types of geometries. MC X-Ray allows for more than 100 different regions in materials with shape of spheres, cylinders and combinations of horizontal and vertical planes. All these regions can have a different composition. MC X-Ray includes the Bremstrallung cross-sections of Ding - Statham to compute the emitted background x-ray intensity in order to generate a synthetic $\mathrm{X}$-Ray spectrum. Then, a true EDS spectrum detected by a SDD detector can be simulated using the absorption of photons and the subsequent diffusion of the photo-electrons in the x-ray detector using the synthetic spectra. MC X-Ray simulates the true noise of EDS spectra without using Gaussian noise techniques, as classically done in others softwares. As a result, complete EDS maps can be simulated for a given number of emitted photons per pixel.

In this work, EDS images were simulated for embedded spheres of $\mathrm{Cu}$ in $\mathrm{C}$ of diameter of 50, 20, 10 and $5 \mathrm{~nm}$ at $10 \mathrm{keV}$ for various number of emitted photons per pixels in $128 \mathrm{X} 128$ images having a size of $140 \times 140 \mathrm{~nm}$. Figure [1] shows such simulated X-Ray images for the C $K \alpha$ and the $\mathrm{Cu} K \alpha$ and $L \alpha$ lines with 1000 and 100000 emitted photons per pixel. At 1000 emitted photons per pixel, all the $\mathrm{Cu}$ spheres are visible for the $\mathrm{C} K \alpha$ and the $\mathrm{Cu} L \alpha$ lines while for the $\mathrm{Cu} K \alpha$ line, the $5 \mathrm{~nm}$ is not visible and the $10 \mathrm{~nm}$ sphere is barely visible. Since at $10 \mathrm{keV}$, the overvoltage is low for the $\mathrm{Cu} K \alpha$ line, even if the x-rays that are coming closer to the surface should give a better spatial resolution in that case, the low ionisation cross-section gives few emitted photons and with a total of 1000 emitted photons per pixels, the small spheres do not emit sufficient photons to be visible. This is not the case with 100000 emitted photons per pixels with all the maps showing the $\mathrm{Cu}$ spheres. In order to quantify the spatial resolution, the Smart routine [2] is used with the simulated x-ray images. Figure [2] shows the resolution of these x-ray images as a function of the total number of emitted photons per pixel. Clearly, the resolution improves with an increase of photon number, especially for the $\mathrm{Cu} K \alpha$ line which has a terrible resolution below 10000 counts. It is interesting to note that images with the $\mathrm{C} K \alpha$ line seem to have the best resolution owing to its stronger absorption.

\section{References}

1. P. Michaud and R. Gauvin (2009), "MC X-Ray, a New Monte Carlo Program for Quantitative X-Ray Microanalysis of Real Materials”, Microscopy and Microanalysis, 15 (Supp.2), p. 488-489.

2. D. C. Joy. The Smart routine is available at http://web.utk.edu/ srcutk/ 


\begin{tabular}{|l|l|l|l|}
\hline $\begin{array}{l}\text { Total number of } \\
\text { emitted photons per } \\
\text { pixel }\end{array}$ & C $K_{\alpha}$ & Cu $L_{\alpha}$ & Cu $K_{\alpha}$ \\
\hline 1000 & & & \\
\hline 100000 & & & \\
\hline
\end{tabular}

Figure [1] Simulated X-Ray images of embedded spheres of $\mathrm{Cu}$ in $\mathrm{C}$ having diameter of 50, 20, 10 and $5 \mathrm{~nm}$ at $10 \mathrm{keV}$ for the $\mathrm{C} K_{\alpha}$ and the $\mathrm{Cu} K_{\alpha}$ and $L_{\alpha}$ lines with 1000 and 100000 emitted photons per pixel. The images have $128 \times 128$ pixels and a size of $140 \times 140 \mathrm{~nm}$.

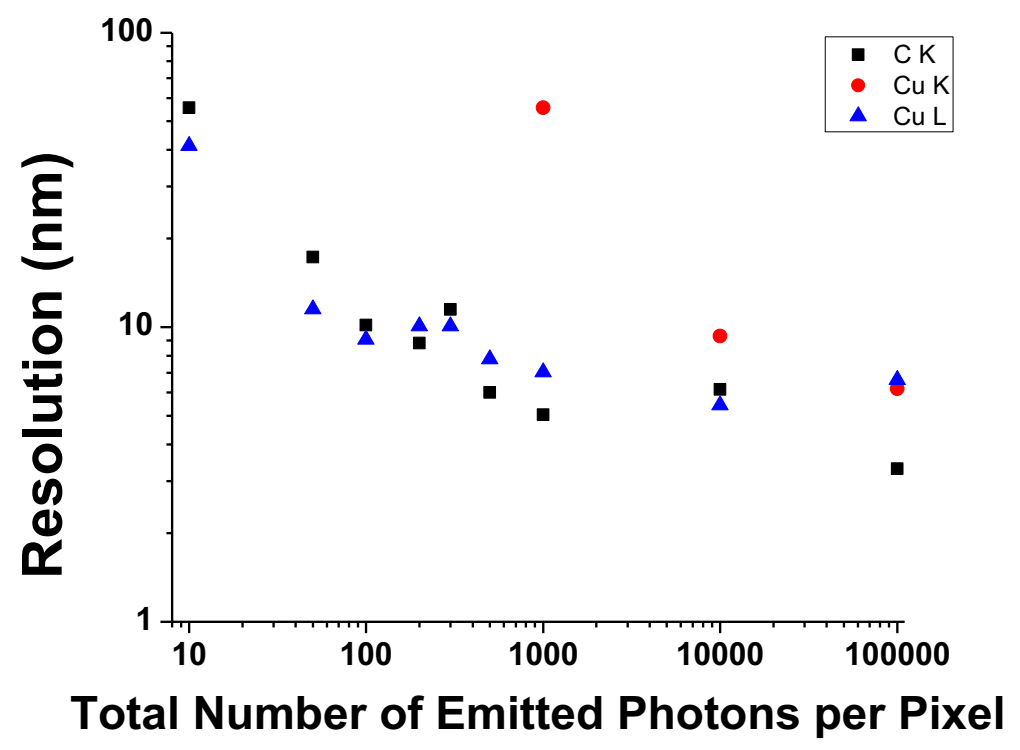

Figure [2] Resolution of the x-ray images as a function of the total number of emitted photons per pixel. 